\title{
The Redshift Evolution of the Stellar Populations in Elliptical Galaxies $^{1}$
}

\author{
Ralf Bender ${ }^{2}$ and Bodo Ziegler ${ }^{2}$ \\ Universitäts-Sternwarte, Scheinerstraße 1, 81679 München, Germany \\ Gustavo Bruzual \\ CIDA, Apartado Postal 264, Mérida 5101-A, Venezuela
}

Received December 1995; accepted 13 march 1996

\footnotetext{
${ }^{1}$ Partly based on observations carried out at the European Southern Observatory, La Silla, Chile.

${ }^{2}$ Visiting Astronomer of the German-Spanish Astronomical Center, Calar Alto, operated by the Max-Planck-Institut für Astronomie, Heidelberg, jointly with the Spanish National Commission for Astronomy
} 


\begin{abstract}
Velocity dispersions $\sigma$ and $\mathrm{Mg}$ absorption line-strengths $\mathrm{Mg}_{b}$ have been measured for a sample of 16 ellipticals in 3 clusters at a redshift of 0.37 . Like local cluster ellipticals, these objects show a correlation between $\operatorname{Mg}_{b}$ and $\sigma$. However, at any given $\sigma$, the mean $\mathrm{Mg}_{b}$ of the ellipticals at $z=0.37$ is weaker than the mean $\mathrm{Mg}_{b}$ of their local relatives in the Coma and Virgo clusters. The $\mathrm{Mg}_{b}$ weakening is smallest for the most luminous ellipticals and larger for the fainter objects. This is unambiguous evidence for small but significant passive evolution of the stellar populations of elliptical galaxies with redshift. It requires that the bulk of the stars in cluster ellipticals has formed at $z>2$. The most luminous objects may even have formed at $z>4$. The $\mathrm{Mg}_{b}-\sigma$ test is a very reliable estimator for the evolution of old stellar populations because it is virtually independent from the stellar initial mass function (IMF) and from the metallicities of the galaxies. Furthermore, the influence of selection effects is minimal.

Consistent with the weakening of $\mathrm{Mg}_{b}$ we find evidence for a B-band luminosity evolution of about $0.5 \pm 0.1 \mathrm{mag}$ at $z=0.37$ from the FaberJackson relation. The combined information about the evolution of $\mathrm{Mg}_{b}$ and luminosity allows us to constrain both the slope of the IMF in ellipticals and the cosmological deceleration parameter $q_{o}$. Our present measurements are compatible with a standard Salpeter IMF and a $q_{o}$ of $0.5 \pm 0.5$.
\end{abstract}

Subject headings: cosmology: observations - galaxies: elliptical and lenticular, $\mathrm{cD}$ - galaxies: evolution - galaxies: formation 


\section{Introduction}

The redshift evolution of galaxies provides strong constraints on their ages and formation processes as well as on theoretical models of structure formation in the Universe. In the specific case of elliptical galaxies, there is the additional prospect that, because of their homogenous properties, it may eventually be possible to calibrate their evolution accurately enough to allow their use as cosmological standard candles or rods.

The luminosity and color evolution of elliptical galaxies or brightest cluster members is generally found to be weak. For various samples of brightest cluster galaxies up to redshifts $z=1$, both optical and infrared colors were found to change only very slowly with $z$ indicating a high redshift of formation (e.g., Aragón-Salamanca et al. 1993, Stanford et al. 1995). Similarly, Hamilton (1985) found only weak evolution in the strength of the $4000 \AA$ break. The bright end of the galaxy luminosity function does not evolve significantly with redshift either, being compatible even with a no-evolution scenario (e.g., Glazebrook et al. 1995, Lilly et al. 1995, Ellis et al. 1996). Observations of distant galaxies up to $z=1$ from the ground and with the Hubble Space Telescope revealed no significant deviations of the surface brightness evolution from the Tolman relation, again an indication of very small if any evolution (Sandage \& Perelmuter 1991, Dickinson 1995, Pahre et al. 1996). At lower redshifts $(z<0.4)$, Franx \& van Dokkum (1996) showed that the mass-to-light ratios of ellipticals as obtained from the fundamental plane relation (Djorgovski \& Davis 1987, Faber et al. 1987) change only very slowly with redshift but in accordance with passive evolution of a very old population.

These results are all indicative of a virtually passive evolution of most cluster ellipticals up to $z \approx 1$. This may be in part a selection effect because ellipticals that experience a merging or accretion event may be transformed into an E+A-type (Dressler \& Gunn 1983) or even bluer object and therefore drop out of an ellipticals sample for some time. But in 
general, these events cannot have injected a significant fraction of young stars into brightest cluster ellipticals below redshifts of 1 . Otherwise, the observed redshift evolution would be much stronger and, also, it would be difficult to explain the high uniformity of colors and absorption line indices in local cluster ellipticals (Bower et al. 1992, Bender, Faber \& Burstein 1993). This does not contradict the claim that many ellipticals were originally formed in major mergers (e.g., Schweizer 1990, Bender 1990b). It simply means that, in rich clusters, the major merging and star formation epoch is to be found at higher redshifts (e.g., Bender, Burstein and Faber 1993, 1994). This picture has recently been shown to be even in agreement with cold dark matter models (Kauffmann 1995).

With the redshift evolution of elliptical galaxies being very weak, the challenge of measuring it accurately is correspondingly bigger. In this paper we will analyse the

evolution of ellipticals with a method that is principally different from the methods applied previously. Our test is based on the tight relation between $\mathrm{Mg}$-Index $\mathrm{Mg}_{b}$ and velocity dispersion $\sigma$ of elliptical galaxies and will be described in Section 2. Section 3 briefly describes sample selection and observations, Section 4 the data analysis. Section 5 presents results and conclusions.

\section{Measuring the evolution of elliptical galaxies with the $\mathbf{M g}_{b}-\sigma$ test}

A reliable measurement of the evolution of elliptical galaxies requires a full understanding of selection effects. This means that the mass and metallicity distributions of the distant galaxy sample as well as of the local comparison sample have to be known very accurately. While the influence of the mass distribution is obvious, the importance of metallicity results from the age-metallicity degeneracy: For stellar populations older than 2 Gyr, an increase in metallicity by a factor of two and a decrease in age by a factor of three results in almost identical optical and near IR colors (Worthey 1994). Therefore, 
the colors of high redshift ellipticals cannot be used to test their evolution as long as their metallicities are not well constrained.

A method that automatically takes into account the effects of different masses and metallicities is given by the $\mathrm{Mg}_{b}-\sigma$ test which we introduce and explain in the following. Nearby cluster ellipticals show a tight correlation between $\mathrm{Mg}_{b}$ and $\sigma$ (e.g., Dressler et al. 1987). Because the $\mathrm{Mg}_{b}$-index depends about equally on metallicity and age, the tightness of the $\mathrm{Mg}-\sigma$ relation implies that, at any given $\sigma$, the combined relative spread in age and metallicity of cluster ellipticals must be smaller than 15\% (Bender, Burstein \& Faber 1993). Therefore, measuring the velocity dispersion of a local elliptical allows to constrain its metallicity to better than 15\%. Moreover, if ellipticals evolve essentially passively below $z \approx 1$, then the same applies to distant ellipticals. I.e., we can assume that all ellipticals up to $z \approx 1$ which have similiar velocity dispersion also have similiar metallicities. Any reduction of $\mathrm{Mg}_{b}$ of distant ellipticals must therefore be due to lower age. Consequently, the observation of the $\mathrm{Mg}-\sigma$ relation as a function of redshift gives a straightforward estimate of the evolution of the stellar populations in elliptical galaxies.

The age determination with the $\mathrm{Mg}-\sigma$ test has the additional advantage that, unlike the evolution of luminosity or surface brightness, the evolution of $M g_{b}$ line strength is virtually independent from the stellar initial mass function (e.g., Worthey 1994). Finally, we note that small differences in the $\mathrm{Mg}-\sigma$ relation which might exist between low and high density environments (e.g., de Carvalho \& Djorgovski 1992, Lucey 1995) do not invalidate our conclusions in the following. This is because the elliptical galaxies we compare at different redshifts all live in the centers of medium rich to rich clusters. 


\section{Sample selection and observations}

The distant elliptical galaxies selected for observation were members of three clusters of galaxies at similar $z$ : Abell $370(z=0.375)$, MS 1512+36 $(z=0.372)$, and CL 0949+44 $(z=0.377)$. The redshift of 0.37 was chosen because it allows a good determination of Mg absorption strengths undisturbed by strong telluric emission and absorption lines. The color selection criterion for the galaxies was $(\mathrm{B}-\mathrm{V})_{\text {restframe }}>0.8$, in turn being equivalent to $\mathrm{Mg}_{\mathrm{b}}>3 \AA$ (using relations between $\mathrm{B}-\mathrm{V}, \mathrm{Mg}_{2}$ and $\mathrm{Mg}_{b}$ from Burstein et al. 1984 and Bender et al. 1993). Colors and magnitudes were taken from Pickles \& van der Kruit (1991) or derived from our own CCD photometry carried out with the Calar Alto 2.2m telescope. Generally, in each cluster the two to four brightest galaxies plus a random sample of fainter objects (obeying however the color constraint) were selected for spectroscopic observation.

The spectroscopy was carried out at the 3.5m telescope on Calar Alto, Spain, and at the ESO NTT on La Silla, Chile, during in total 26 nights between December 1993 and July 1995. We used the Boller \& Chivens double spectrograph at the Calar Alto and the EMMI focal reducer at ESO. The redshifted galaxies and the local template stars were observed in the same rest wavelength range between about $4500 \AA$ to $6000 \AA$ and with identical spectral resolution $\left(\sigma_{\text {instr }} \approx 100 \mathrm{~km} / \mathrm{s}\right)$. The aperture size was $3.5 "$ by $3.5 "$. Exposure times between 8 and 12 hours were needed to reach a signal-to-noise ratio of at least 40 per $\AA$ in the spectra of the galaxies.

\section{Data analysis}

Spectroscopy. The CCD spectra were reduced in the standard way (e.g., Bender, Saglia \& Gerhard 1994). Special care was taken to achieve accurate sky subtraction. The flux standards were used to control the strength of weak telluric $\mathrm{H}_{2} \mathrm{O}$ absorption bands 
around $7200 \AA$. The velocity dispersions of the galaxies were derived using the Fourier Correlation Quotient method (Bender 1990a). The $\mathrm{Mg}_{b}$ equivalent width was determined as described in Faber et al. (1985). $\mathrm{Mg}_{b}$ was corrected for differences in spectral resolution, for velocity dispersion broadening and for redshift dependent factors. Because the spectra of the $z=0.37$ ellipticals were integrated over a much larger physical surface area than the spectra of the nearby comparison ellipticals in the Coma and Virgo cluster (taken from Dressler et al. 1987), an aperture correction had to be applied. We zeroed the correction to a 4" by 4" projected aperture size at the distance of the Coma Cluster. The aperture correction was computed from velocity dispersion profiles and $\mathrm{Mg}_{b}$ profiles published in, e.g., Davies et al. (1993), Gonzalez (1993), Carollo et al. (1995) and Fisher et al. (1995).

Finally, the $\mathrm{Mg}_{2}$ absorption magnitudes of the local comparison sample of cluster ellipticals in Coma and Virgo (Dressler et al. 1987) had to be transformed to $\mathrm{Mg}_{b}$ equivalent widths because for the distant ellipticals the $\mathrm{Mg}_{b}$ values could be determined with much better $\mathrm{S} / \mathrm{N}$ than $\mathrm{Mg}_{2}$ values. For that purpose we used the very good $\mathrm{Mg}_{b}-\mathrm{Mg}_{2}$ correlation, empirically given in Burstein et al. (1984) and Gonzalez (1993).

Imaging. In order to analyse the Faber-Jackson relation for the redshifted ellipticals, their rest-frame total luminosities in the B-band had to be calculated. For this purpose, we first extrapolated the observed BVRI aperture magnitudes to total magnitudes on the basis of the ratio between the galaxies' effective radii $r_{e}$ and the aperture size. An estimate of the $r_{e}$ was obtained using the $r_{e}-\sigma$ relation of nearby ellipticals. Typical aperture corrections ranged between 0.2 and $1.1 \mathrm{mag}$, with an error of about $0.2 \mathrm{mag}$ (as estimated from the scatter of the local $r_{e}-\sigma$ relation). In the second step, rest-frame $B$ luminosities were calculated from fits of spectral energy distributions (from Bruzual \& Charlot 1996) to the observed BVRI values. 


\section{Results and Discussion}

Figure 1 shows the $\mathrm{Mg}_{b}-\sigma$ relation for cluster ellipticals at a redshift of $z=0.37$ in comparison to local elliptical galaxies in the Coma and Virgo cluster. Two main conclusions can be drawn:

\section{EDITOR: PLACE FIGURE 1 HERE.}

(a) The distant ellipticals also show a correlation between $\mathrm{Mg}_{b}$ index and velocity dispersion $\sigma$ as local ellipticals do. The slope of the $\mathrm{Mg}_{b}-\sigma$ relation at higher redshift appears to be slightly steeper than today indicating that low luminosity ellipticals may be systematically younger than high luminosity ellipticals. This may support a recent claim by Faber et al. (1995) according to which the mean ages of ellipticals should systematically decrease with decreasing luminosity. The distant objects with very low $\mathrm{Mg}_{b}$ could be genuinely younger or may only have experienced a more recent but smaller star formation event.

(b) There is clear evidence for evolution: at any given velocity dispersion, the mean $\mathrm{Mg}_{b}$ of ellipticals at $z=0.37$ is significantly weaker than at $z=0$. The effect is relatively weak for big ellipticals and larger for small ellipticals. On average, the $\mathrm{Mg}_{b}$ absorption is reduced by about $0.3 \AA$. Note that this conclusion is not affected by selection biases since our color selection criterion (see Section 3) only cuts off ellipticals with $\mathrm{Mg}_{b}<3 \AA$.

On the basis of Worthey's (1994) population synthesis models we can use this weaking of $\mathrm{Mg}_{b}$ to estimate the ratio of the mean age of the $z=0.37$ ellipticals relative to the mean age of local ellipticals. We derive the following dependence of $\mathrm{Mg}_{b}$ on age $t$ and metallicity $Z: \log \mathrm{Mg}_{b}=0.37+0.20 \log t+0.31 \log Z / Z_{o}\left(\right.$ valid for $t>5$ Gyrs and $1 / 3<Z / Z_{\odot}<3$ ). The proportionality factors of $\log t$ and $\log Z$ are very robust and only weakly dependent 
on IMF and on uncertainties in the population synthesis models (Bruzual 1996). The small evolution we see in Figure 1 strongly supports the notion that ellipticals have evolved mostly passively between $z=0.37$ and $z=0$. Since then $Z \approx$ const., the observed reduction of $\mathrm{Mg}_{b}$ can be directly translated into a relative age difference. The three dashed lines in Figure 1 give the expected $\mathrm{Mg}_{b}-\sigma$ relation at $z=0.37$, if the mean formation redshifts of the stars in ellipticals were formed at $z_{f}=4.5,2,1$, respectively. Independent from $\mathrm{H}_{o}$ and only weakly dependent on $\mathrm{q}_{o}$ we conclude that the bulk of the stars in the luminous cluster ellipticals must have formed at redshifts $\mathrm{z}>\mathbf{2}$, the higher luminosity objects may even have formed at $\mathbf{z}>4$. Because of the possible presence of some younger stars injected in minor accretion events and because we observe luminosity-weighted spectra, these age estimate is rather a lower limit.

\section{EDITOR: PLACE FIGURE 2 HERE.}

The age-driven reduction of the $\mathrm{Mg}_{b}$ absorption in ellipticals at higher redshifts should correspond to an increase in their luminosity. This should be detectable via the Faber-Jackson relation (Faber \& Jackson 1976). In order to derive the Faber-Jackson

relation for our distant cluster ellipticals, their rest-frame $B$ luminosities were calculated as described in Section 4 and using $H_{o}=50 \mathrm{~km} / \mathrm{s} / \mathrm{Mpc}$ and $q_{o}=0.5$.

The Faber-Jackson relation for the $z=0.37$ cluster ellipticals and for the Coma and Virgo ellipticals is shown in the upper panel of Figure 2. It is evident that the distant ellipticals are brighter than their local counter-parts by about $0.5 \pm 0.1$ mag in the B-band. Is this consistent with the weakening of $\mathrm{Mg}_{b}$ in the $\mathrm{Mg}-\sigma$ relation? Using Worthey's models with a Salpeter IMF, we obtain that age variations lead to a correlated change of the B-band luminosity with the $\mathrm{Mg}_{b}$ value according to $\Delta=1.2 \Delta \mathrm{Mg}_{\mathrm{b}} / \AA$. Thus, the evolution of the $\mathrm{B}$ luminosity as estimated from the weakening of $\mathrm{Mg}_{b}$ should be $\Delta B(z \approx 0.4) \approx-0.4$ 
mag, consistent with the actually observed brightening. This corresponds to an evolution in rest frame $(B-V)$ of $-0.04 \mathrm{mag}$ and $(V-K)$ of $-0.12 \mathrm{mag}$, in accordance with the measurements of Stanford et al. (1995).

We can also turn the above procedure around and correct the luminosity of each galaxy by its individually calculated evolution correction $\Delta \mathrm{M}_{\mathrm{B}}=1.2 \Delta \mathrm{Mg}_{\mathrm{b}} / \AA$. The lower panel of Figure 2 shows the result: the $z=0.37$ ellipticals and the Coma and Virgo ellipticals now all fall on top of each other. Even the slope of the Faber-Jackson relation at $z=0.37$ is similar within the errors to the locally observed slope.

We conclude that the evolution of the stellar populations in ellipticals as derived from the $\mathrm{Mg}_{b}-\sigma$ relation and from the Faber-Jackson relation are consistent. Within the limits of our errors, there is no evidence for a very unusual slope of the IMF, nor a very unusual value of the cosmological constant. Changing the slope of the IMF by $\Delta x=1$ would cause a change of the luminosities at $z=0.37$ by ca. \pm 0.13 mag (Bruzual \& Charlot 1996 models). Similarly, changing $q_{o}=0.5$ by \pm 0.5 causes a change of the luminosities by ca. $\pm 0.11 \mathrm{mag}$.

In a future paper, we will combine our spectroscopic data with HST photometry. This will allow us to investigate the evolution of the elliptical galaxies more accurately by employing the fundamental plane relations. In combination with the Tolman surface brightness test, one may then also be able to obtain better and, more importantly, independent constraints on the slope of the IMF and on the value of $q_{o}$.

We thank the Calar Alto and La Silla staff for efficient support during the observations. Discussions with Drs. P. Belloni, L. Greggio, U. Hopp, G. Kauffmann, A. Renzini and R. Saglia are gratefully acknowledged. Thanks also go to Dr. R. Carlberg who provided us with a list of red members of MS 1512+36. The work of R.B. and B.Z. was supported by SFB 375 of the DFG and by the MPG. 


\section{REFERENCES}

Aragón-Salamanca, A., Ellis, R.S., Couch, W.J. \& Carter, D. 1993, MNRAS, 262, 764

Bender, R. 1990a, A\&A, 229, 441

Bender, R. 1990b, in Dynamics and Interactions of Galaxies, ed. R. Wielen, Springer Verlag, Berlin, 232

Bender, R., Burstein, D. \& Faber, S. 1993, ApJ, 411, 153

Bender, R., Burstein, D. \& Faber, S. 1994, in Panchromatic View on Galaxies, eds. G. Hensler et al., Editions Frontières, Gif-sur-Yvette

Bender, R., Saglia, R. P., \& Gerhard, O. E. 1994, MNRAS, 269, 785

Bower, R., Lucey, J.R. \& Ellis, R.S., 1992, MNRAS, 254, 601

Burstein, D., Faber, S.M., Gaskell, C.M. \& Krumm, N. 1984, ApJ, 399, 462

Bruzual, G. 1996, in IAU Symp. 171, eds. Bender, R. \& Davies, R. L., Kluwer, Dordrecht

Bruzual, G. \& Charlot, S. 1996, ApJ, in preparation

Carollo,C. M., Danziger, I. J. \& Buson, L. 1995, MNRAS 265, 553

Davies, R.L., Sadler, E. \& Peletier, R. 1993, MNRAS, 262, 650

de Carvalho, R.R. \& Djorgovski, S. 1992, ApJ, 389, L49

Dickinson, M. 1995, in ASP Conf. Ser. 86, Fresh Views of Elliptical Galaxies, eds. A. Buzzoni et al., 283

Djorgovski, S. \& Davis, M. 1987, ApJ, 313, 59

Dressler, A., Gunn, J. 1983, ApJ 270, 7 
Dressler, A., Lynden-Bell, D., Burstein, D., Davies, R.L., Faber, S.M., Terlevich, R. \& Wegner, G. 1987, ApJ, 313, 42

Ellis, R. S., Colless, M., Broadhurst, T., Heyl, J. \& Glazebrook, K. 1996, MNRAS, in press

Faber, S. M., Dressler, A., Davies, R. L., Burstein, D., Lynden-Bell, D., Terlevich, R. \& Wegner, G. 1987, in Nearly Normal Galaxies, From the Planck Time to the Present, ed. S. M. Faber, Springer New York, 175

Faber, S. M., Friel, E. D., Burstein, D. \& Gaskell, C. M. 1985, ApJS, 57, 711

Faber, S. M. \& Jackson, R. E. 1976, ApJ, 204, 668

Faber, S. M., Trager, S. C., Gonzalez, J. J. \& Worthey, G. 1995, in IAU Symp. 164, eds. van der Kruit, P. C. \& Gilmore, G., Kluwer, Dordrecht, 249

Fisher, D., Illingworth, G. \& Franx, M. 1995, ApJ, 438, 539

Franx, M. \& van Dokkum, P. G. 1996, in IAU Symp. 171, eds. Bender, R. \& Davies, R. L., Kluwer, Dordrecht

Glazebrook, K., Peacock, J. A., Miller, L. \& Collins, C. A. 1995, MNRAS, 275, 169

Gonzalez, J. 1993, PhD Thesis, University of California at Santa Cruz

Hamilton, D. 1985, ApJ, 297, 371

Lilly, S. J., Tresse, L., Hammer, F., Crampton, D. \& LeFevre, O. 1995, ApJ, 455, 108

Lucey, J. R. 1995, in IAU Symp. 164, ed. van der Kruit, P. C. \& Gilmore, G., Kluwer, Dordrecht, 281

Kauffmann, G. 1996, MNRAS, in press

Pahre, M.A., Djorgovski, S.G., de Carvalho, R.R. 1996, ApJ Letters, in press 
Pickles, A. J. \& van der Kruit, P. C. 1991, A\&AS, 91, 1

Sandage, A. \& Perelmuter, J-M. 1991, ApJ, 370, 455

Schweizer, F. 1990, in Dynamics and Interactions of Galaxies, ed. R. Wielen, Springer Verlag, Berlin, 60

Stanford, S. A., Eisenhardt, P. \& Dickinson, M. 1995, ApJ, 450, 512

Worthey, G. 1994, ApJS, 95, 107 
Fig. 1. $-\mathrm{Mg}_{b}-\sigma$ relation for ellipticals in the Coma and Virgo cluster (small open circles, typical error bar in the lower right corner) and for ellipticals at $z=0.37$ in Abell 370 (squares), MS 1512+36 (triangles) and CL 0949+44 (circles). For the latter, filled symbols refer to reliable measurements within the errorbars, open symbols to systematic uncertainties larger than the error bars and due to emission lines and/or night sky subtraction problems. The solid line is a fit to the Virgo and Coma ellipticals, the dashed lines correspond to the expected $\mathrm{Mg}_{b}-\sigma$ relation at $z=0.37$ for a mean formation redshift of the stars in ellipticals at $z_{f}=4.5,2,1$ (top to bottom; $q_{o}=0.5$ ). The arrow in the lower right corner gives the size of the aperture correction that has been applied to the distant ellipticals.

Fig. 2.- The Faber-Jackson relation for Coma and Virgo ellipticals (small dots) and for ellipticals at $z=0.37$ ( $q_{o}=0.5$, symbols as in FIG. 1$)$; upper panel: rest-frame $\mathrm{M}_{B}$, lower panel: $\mathrm{M}_{B}$ of $z=0.37$ ellipticals corrected for evolution (see text for explanation). The solid line is a fit to the Virgo and Coma ellipticals. 


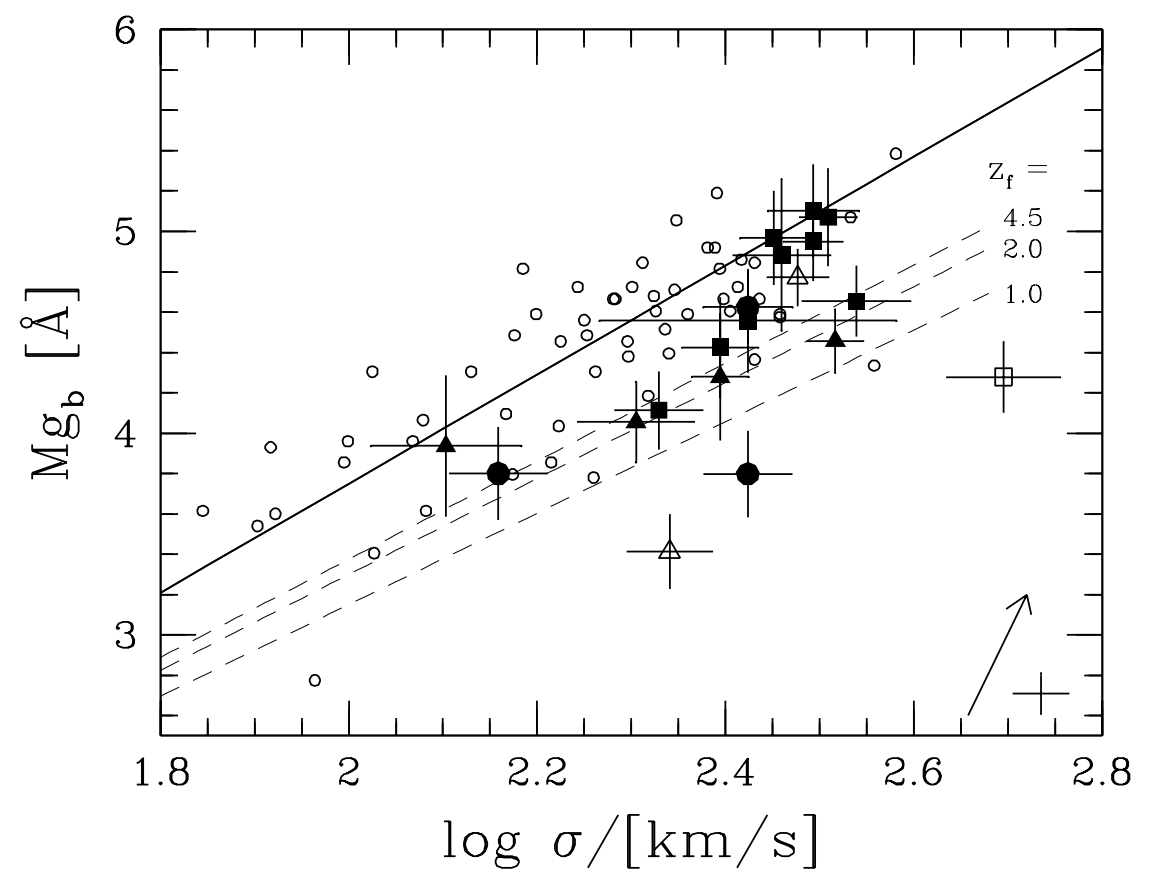




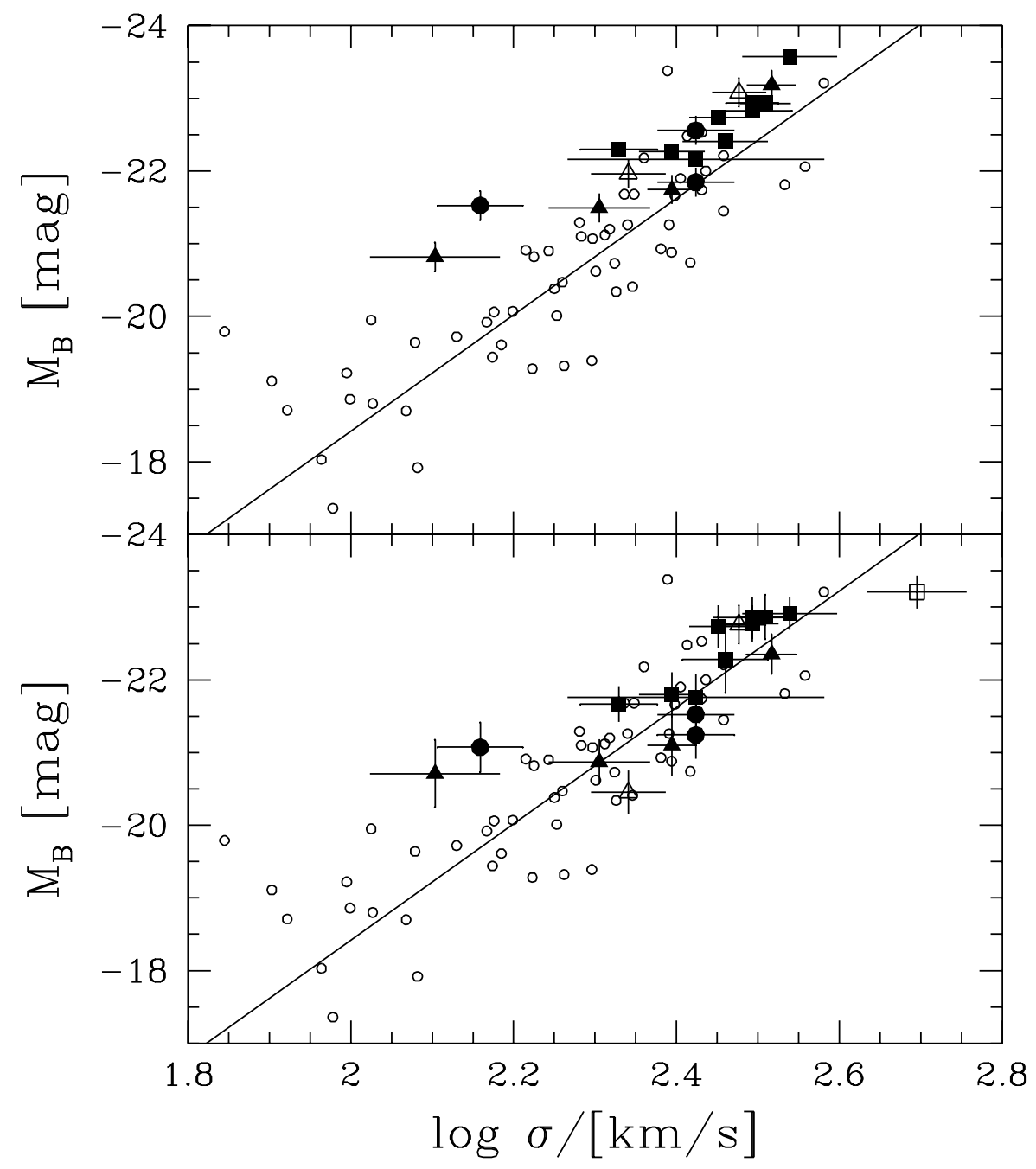

